\title{
The Dynamic Characteristics of the Controllable Floating Raft System
}

\author{
Chao Geng ${ }^{1}$, Zeyu Weng ${ }^{1}$, Hui Wang ${ }^{2}$ and Jie Tang ${ }^{1}$ \\ ${ }^{1}$ College of Mechanical Engineering, Zhejiang University of Technology, Hangzhou 310014, China \\ ${ }^{2}$ HangzhouPopwilInstrument Co., Ltd, Hangzhou 310014, China
}

\begin{abstract}
In this paper, the dynamic characteristics of some controllable floating raft test system are studied. The virtual dynamic model of the system is established in Adams, the acceleration responses of system in different damping states are simulated, and the vibration level differences of system in different damping states are contrasted. The results show that it is able to improve the vibration isolation property in wide frequency range by switch on-off control of the controllable damper. Finally the possibility to improve the vibration isolation property of system by semi-active control of variable damper is discussed.
\end{abstract}

\section{Keywords-floating raft; vibration isolation; semi-active control}

\section{INTRODUCTION}

The floating raft is a vibration isolation device installed on ships. In floating raft system, multiple machines as vibration sources are elastically installed on same raft, and then the raft is resiliently mounted on the ship. By the design of elastic isolators and middle raft in system, the vibration of resources passed to the ship can be effectively weakened [1].Many scholars have done a lot of research on the vibration isolation property and structure design of the floating raft system [2][3].

The structure parameters damping, stiffness and mass of floating raft installed on ships can't be adjusted, once the floating raft system is installed on the ship, the vibration isolation property of system is fixed, which is not so satisfying in some frequency range, especially near resonant frequencies. With the development of control technology, active control and semi-active control are introduced to vibration isolation systems. The semi-active control is actually a type of structure parameters control. By installing controllable isolators whose stiffness or damping can be changed in the vibration system, controllable vibration isolation system could be built. It is able to adopt the isolation property of controllable vibration isolation system to vibration sources by controlling the controllable isolators. Compared with active control, the semi-active control is easier to realize, its energy consumption is lower, and its control quantity is close to the active control, which has good prospects of application. There are already some research on the semi-active control of vibration isolation system of vehicle suspensions and high buildings, but the study on semi-active control of floating raft system is rare now. Scholars from Shanghai Jiaotong University studied the semi-active fuzzy control of floating raft system, but the study is based on some high quality dampers, and the control is relatively complex which limit its application [4][5].
Zhejiang University of Technology invents a kind of variable damper controlled by switch. When the switch is in on state, the damper is energized, the damping of the damper is high, when the switch is in off state, the damper isn't energized, the damping become low, and the damping states of this variable damper could be switched quickly. By installing these dampers in the floating raft system, Zhejiang University of Technology built a controllable floating raft test system whose dynamic characteristics could be adjusted. The study in this paper is based on this floating raft test system, which has not been studied yet from the documents published now.

In this paper, the virtual dynamic model of this controllable floating raft test system is established in Adams, the dynamic characteristics of the system indifferent damping states are simulated, and the method to improve the vibration isolation property of system by switch on-off control are probed.

\section{EVALUATION OF VIBRATION ISOLATION PROPERTY}

The main purpose of designing vibration isolation system is try to increase the vibration isolation property. In actual design, an appropriate evaluation index which could exactly reflect the reduction of vibration passed to isolation object is very important, and it's the base of analyzing and designing the system. The main evaluation indexes used now include force transmissibility, insertion loss, vibration level difference and power flow.

Force transmissibility is the primary evaluation index, which is the ratio of force passed to vibration isolation target to excitation force. Force transmissibility can directly reflect the isolation property of system. In practice, the excitation force and the force passed to the target are hard to measured, and the real force transmissibility is hard to gotten. Force transmissibility is mainly used in early design to analyze and predict the isolation property in theory, Insertion loss is the ratio of isolation object response without isolation system to response installing isolation system. The insertion loss is usually measured by test, which can well reflect the real isolation property of system, but in most actual projects, it is impossible to remove the isolation system and test the response without isolation system. The power flow doesn't use force or displacement to evaluate the isolation property, it uses the vector product of force and velocity to study the vibration transmission in system, the power flow could reflect the work done by excitation force and energy consumed by isolation system in unit time. The power flow is seldom be used to evaluate the isolation property of system in practice cause the 
measurement of power flow is complex. The vibration level difference (VLD) is the ratio of vibration source response to vibration isolation object response. $\mathrm{T}$ he response could be displacement, velocity or acceleration. Compared with the other evaluation indexes of vibration isolation property, the displacement, velocity and acceleration are easy to be measured by sensors, and the VLD is easy to be gotten. The VLD has been widely used to evaluate the isolation property of isolation system installed on ships. In this paper, the acceleration VLD is chosen to evaluate the isolation property of system. The acceleration $\mathrm{VLD} L_{A}$ can be expressed by

$$
\mathrm{L}_{\mathrm{A}}=20 \log \left(\frac{\ddot{\mathrm{x}}_{1}}{\ddot{\mathrm{x}}_{2}}\right)
$$

Where $\ddot{x}_{1}$ is acceleration responseof vibration source, $\ddot{x}_{2}$ is the acceleration response ofisolation object.

\section{VIRTUAL MODEL OF CONTROLLABLE FLOATING RAFT SYSTEM}

The simulation is based on the actual test system. Main structure of test system includes vibration source, machine, raft, foundation, springs, controllable dampers and oriented pillars. The structure of test system is as Figure I

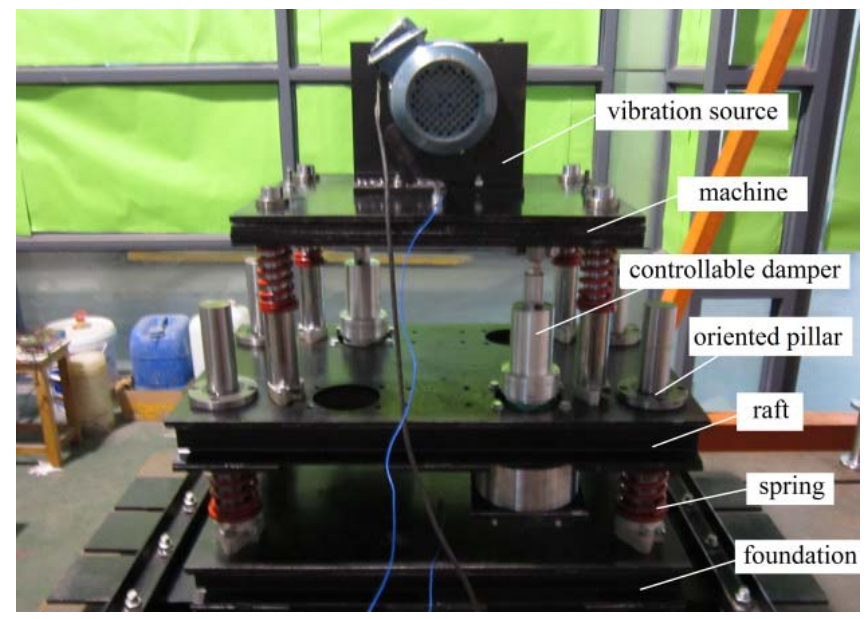

FIGURE I. CONTROLLABLE FLOATING RAFT TEST SYSTEM

The vibration resource and machine are fixed together to simulate real motor installed on ship, the machine is composed of four steel plates. Two controllable dampers are installed between the machine and raft, which are controlled by switches. The vibration source of the actual test system is composed of a pair of symmetric gears. The gears are with eccentric blocks on them and be driven by electric motor. The structure of vibration source is as Figure II.

The centrifugal forces of two eccentric blocks are respectively $F_{1}$ and $F_{2}$, which balance in the horizontal direction, and the resultant force of $F_{1}$ and $F_{2}$ in the vertical direction form a sine force $F$, the amplitude of vertical centrifugal force is related to the revolving speed of motor. The amplitude of vertical centrifugal force can be calculated by

$$
f=1.776 \omega^{2}
$$

Where $f$ is the amplitude of vertical force $F$, unit $\mathrm{N}$; $\omega$ is the frequency of $F$, unit $\mathrm{Hz}$, which is the same as the revolving speed of motor.

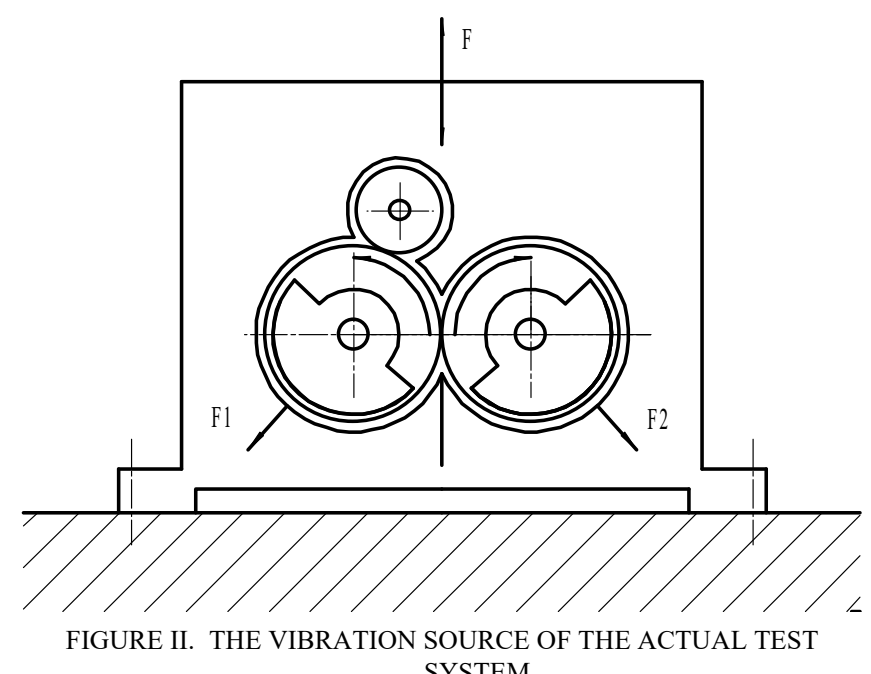

SYSTEM

Establish the virtual model of the controllable floating raft system in Adams, the model established in Adams includes the machine, raft, foundation and spring components.

The spring components are used to simulate the springs and dampers, set two spring components in vertical direction between the machine and raft to simulate the springs and dampers upper, set four spring components between the raft and foundation to simulate the springs down. The stiffness of the machine and raft are very high, whose deformation are much less comparing with the vibration response of the system, so both two of them are established as rigid bodies in Adams. Establish the model of machine, and set the mass of the machine as the total mass of the vibration source and machine, because the vibration source and machine in actual test system are united together. The oriented pillars above are fixed on the raft, so the oriented pillars above and the raft should be established as a whole, and the mass should be set as the sum of them. The foundation of the actual test system is soft in vertical direction, which could be established as a rigid body supported by springs and dampers. The oriented pillars under are fixed on the foundation, so establish the pillars under and the foundation as a whole body too. Set four spring components in vertical direction between the foundation and ground to simulate the equivalent stiffness and damping of the foundation. The oriented pillars constrain the movements of the system to vertical direction, so constrain the motion of the machine, the raft and the foundation to vertical direction in Adams. The simulation model established in Adams/view is as Figure III. 


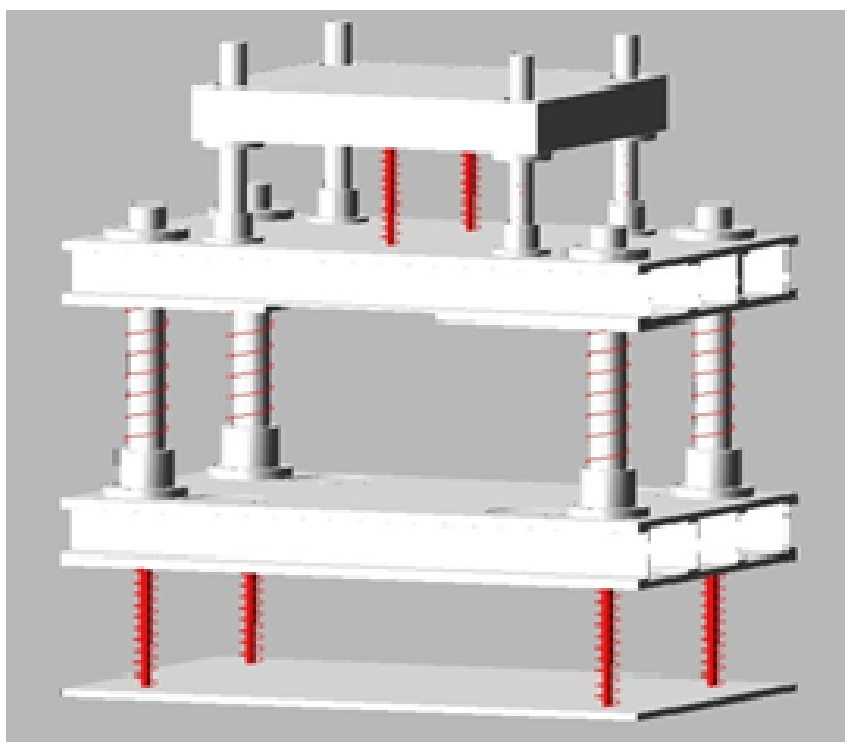

FIGURE III. THE VIRTUAL MODEL OF CONTROLLABLE FLOATING RAFT SYSTEM

\section{The Simulation of the Controllable FloAting RAFT SYSTEM}

Use the Adams/vibration block to simulate the acceleration responses of the machine and foundation, which could analyze the dynamic response of the system under different excitation

ntrollable damper as the low damping and simulate the machine and foundation acceleration frequency responses in switch off state which is as Figure IV. Set the damping of the controllable damper as the high damping and simulate the machine and foundation frequency acceleration responses in switch on state which is as Figure V.

The acceleration frequency responses show that the acceleration passed to foundation form machine in two different switch states are obviously weakened. The acceleration frequency response curves of machine and foundation in different states exist some peaks, at peaks the acceleration response are larger.

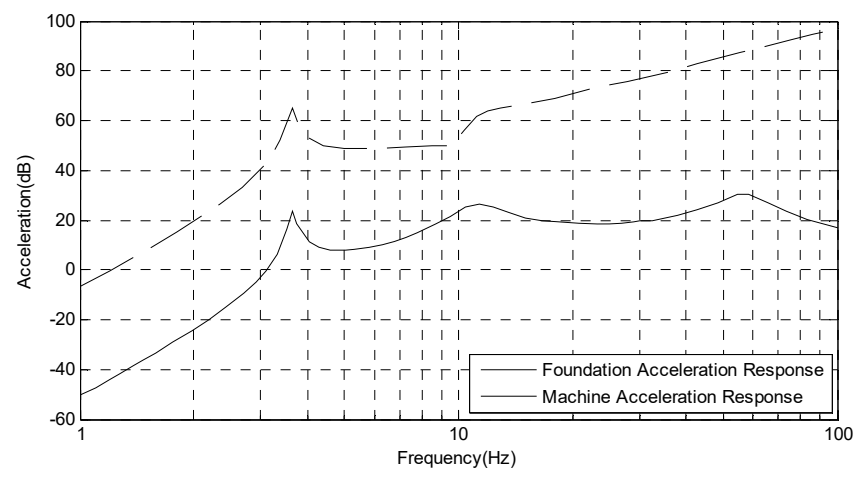

FIGURE IV. ACCELERATION FREQUENCY RESPONSESIN ON STATE force in some frequency range.

The structural parameters in simulation model are set as the actual parameters of controllable floating raft test system. The parameters are as follow:

The total mass of the vibration source and the machine is $233 \mathrm{~kg}$, the total mass of the raft and oriented pillars upper is $185 \mathrm{~kg}$, the total mass of the foundation and the oriented pillars down is $200 \mathrm{~kg}$, the total stiffness of the springs between the machine and the raft is $406400 \mathrm{~N} / \mathrm{m}$, the total damping is $70 \mathrm{~N} /(\mathrm{m} / \mathrm{s})$, the total stiffness of springs between raft and foundation is $281000 \mathrm{~N} / \mathrm{m}$, the total damping is $50 \mathrm{~N} /(\mathrm{m} / \mathrm{s})$, when the switch is in on state, the total damping of the controllable damper is $50000 \mathrm{~N} /(\mathrm{m} / \mathrm{s})$, when the switch is in off state, the total damping of the controllable damper is $1600 \mathrm{~N} /(\mathrm{m} / \mathrm{s})$, the equivalent stiffness of the foundation in the vertical direction is $25320000 \mathrm{~N} / \mathrm{m}$, the equivalent damping of the foundation in the vertical direction is $5000 \mathrm{~N} /(\mathrm{m} / \mathrm{s})$.

Apply the excitation force on the machine in vertical direction, which is set as the actual excitation force expressed by (2). The isolation property of the floating raft system in low frequency range is more important, set the simulation frequency as $1-100 \mathrm{~Hz}$.

Simulate the dynamic characteristics of the controllable floating raft system in different switch states, get the machine and foundation acceleration frequency responses in different switch states. Set the damping of the co

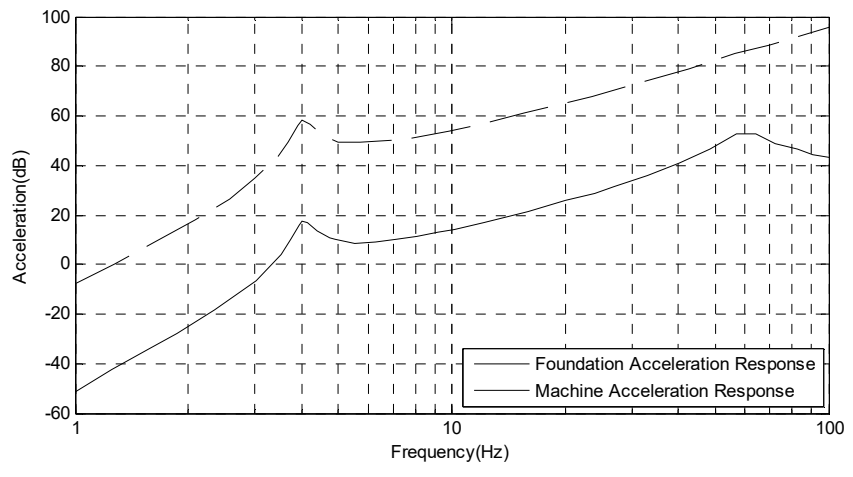

FIGURE V. ACCELERATION FREQUENCY RESPONSESIN ON STATE

In switch off state, the acceleration frequency response of machine exists two peaks, the corresponding frequencies are $3.63 \mathrm{~Hz}$ and $11.48 \mathrm{~Hz}$; the acceleration frequency response of foundation exists three peaks, the corresponding frequencies are $3.63 \mathrm{~Hz}, 11.48 \mathrm{~Hz}$ and $57.54 \mathrm{~Hz}$. The acceleration response of the machine increases with the frequency after the first peak, the response of foundation is not obviously increased with the frequency after the first peak.

In switch on state, the acceleration frequency response of machine exists only one peak, the corresponding frequency is $3.98 \mathrm{~Hz}$; the acceleration frequency response of foundation exists two peaks, the corresponding frequencies are $3.9 \mathrm{~Hz}$ and $58.94 \mathrm{~Hz}$. The acceleration response of the machine increases with the frequency, the response of foundation increases with the frequency first and then decrease after the second peak frequency. 
The amplitude of excitation force is not constant but increases with the frequency, so the acceleration response of the system can't directly reflect the isolation property, use the acceleration VLD to analyze the isolation property. The acceleration VLD of the controllable floating raft system indifferent switch states in frequency range $1-100 \mathrm{~Hz}$ can be calculated from the acceleration frequency responses. The acceleration VLD calculated is as Figure VI.

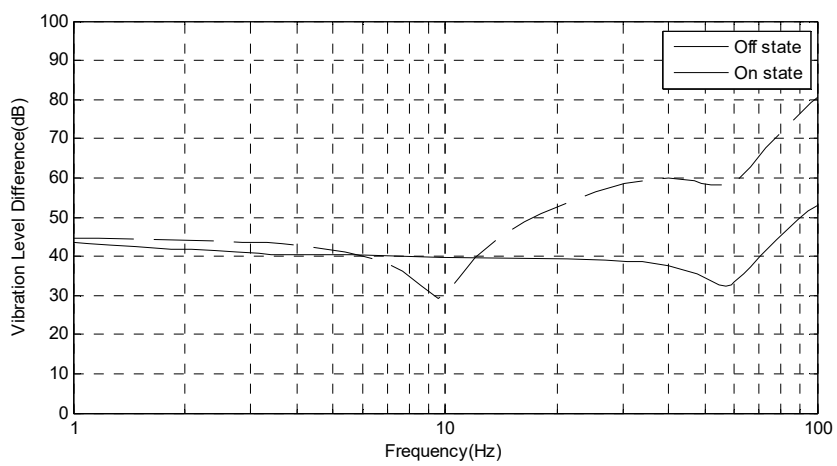

FIGURE VI. ACCELERATIONVIBRATION LEVEL DIFFERENCES

The acceleration VLD in different switch states are different. In low frequency range, the acceleration VLD in switch off state and on state are very near, but in high frequency range, the acceleration VLD are quite different.

In the switch off state, the VLD curve has two distinct wave troughs, the frequency of first trough is near $10 \mathrm{~Hz}$, the second is near $58 \mathrm{~Hz}$, the trough frequencies are respectively the first and second natural frequencies in off state. The acceleration VLD are smaller at the natural frequencies, at the first natural frequency near $10 \mathrm{~Hz}$, the isolation property is worst, the value of VLD is about $29 \mathrm{~dB}$, after the second natural frequency, the acceleration VLD increase quickly with the frequency.

In switch on state, there is also one obvious wave trough near $58 \mathrm{~Hz}$, which is the first natural frequency in on state. At the first natural frequency, the isolation property is worst, the value of the acceleration VLD is about $32 \mathrm{~dB}$, before the first natural frequency, the acceleration VLD is near $40 \mathrm{~dB}$, after the first natural frequency, the VLD also obviously increase with the frequency.

In most frequency range between $1-100 \mathrm{~Hz}$, especially in high frequency range, the acceleration VLD in off state is larger than on state, it means the vibration isolation property in switch off state is generally better than the on state. At the frequencies near $10 \mathrm{~Hz}$, the isolation property in off state is worse than on state. At the frequencies near $58 \mathrm{~Hz}$, the isolation properties of both the on state and the off state become worse, but the isolation property in off state is still better than on state.

So it's feasible to improve the isolation property of controllable floating raft system by switch on-off control of the dampers states. We can easily give a switch on-off control method according to the acceleration VLD. Depending on the frequency of the excitation force, in most frequency range, keep the state of switch in off state, when the frequency of the excitation force is between $6.02 \mathrm{~Hz}$ and $12.02 \mathrm{~Hz}$,turn the switch to on state, the vibration isolation property will be better, and the isolation property in whole frequency range will be improved.

The switch on-off control method given above just chooses the better isolation property in two states at the excitation frequency. The acceleration response gotten by simulation is in fact the response amplitude of the system at the stable states, the acceleration VLD reflect the isolation property at stable states. It is able to further improve the isolation property by the time domain analysis of the system. Depending on the states of the system and the motivation force, it is possible to provide some control strategy, control the damping force between the machine and raft, suppress the vibration of the foundation, and realize the semi-active control of the controllable floating raft system in time domain.

\section{CONCLUSION}

The Adams simulation results show that it is possible to improve the vibration isolation property of the controllable floating raft system in the whole frequency range by switch on-off control of the controllable dampers. The vibration isolation property of the system could be further improved by realizing the on-off semi-active switch control of the floating raft system.

\section{REFERENCES}

[1] ChengZhao, Dayue Chen, "Control for Floating Raft Isolation System," China Mechanical Engineering.China, vol. 19, No. 3, pp. 253-257, Feburary2008.

[2] JikuangYan, Miqun Shen, Guoqing Shang, "The selection of floating raft dynamic parameters" ,Noise and Vibration Control. China, vol. 01, pp.2-9, May 1995.

[3] HualiangZhang, Zuqing Qu, Zhifang Fu, "The effect of main structure parameters on floating raft vibration isolation property", Journal of vibration and shock. China,vol. 19, No. 3, pp.5-8, June 2000.

[4] ChengZhao, Dayue Chen, "Semi-active Fuzzy Sliding Mode Control for Floating Raft Isolation System", Chinese Journal of Mechanical Engineering. China, vol. 44, No. 2, pp.163-169, Feburary2008.

[5] ChengZhao, "Semi-active variable structure control for floating raft isolation system", Journal of Ship Mechanics. China, vol. 18, No.11, pp.1367-1376. November 2014. 